\title{
Multi-mode Convergence-based Indoor Wireless Positioning System Design
}

\author{
Shuang $\mathrm{Xu}^{1, \mathrm{a}}$, Zhigang $\mathrm{Wen}^{1,2, \mathrm{~b}}$ \\ ${ }^{1}$ School of Electronic Engineering, Beijing University of Posts and Telecommunication, Beijing, \\ 100876, China \\ ${ }^{2}$ Institute of Sensing Technology and Business, BUPT, Wuxi, 214000, China \\ aemail: sdlwxs@126.com, bemail: zwen@bupt.edu.cn
}

Keywords: Multimode Fusion; Indoor Positioning Method; Reliability

\begin{abstract}
In this paper, an indoor positioning method is presented for self-localization of a mobile device by combining Wi-Fi wireless location, strap-down inertial navigation systems (SINS), augment reality and ultrasonic positioning. Over the last few years, inertial sensors with low cost and mobile devices equipped with a camera and microphone have become available. Although inertial sensors exhibit large errors, augment reality and ultrasonic positioning can be used to correct the drift error based on this technology. On the other hand, INS sensors can interact with the Wi-Fi positioning system as they provide high-accuracy real-time navigation. The localization algorithm based on reliability for a variety of information fusion processing is proposed to adapt different indoor environment. Finally, the benefits of the proposed architecture are evaluated and compared with the pure Wi-Fi positioning method.
\end{abstract}

\section{Introduction}

Location-based service (LBS) [1] [2] is very meaningful for people's life. The core technology of LBS is positioning, the application of positioning technology with high precision can provide people with more reliable and more practical services such as navigation path, which has very good practical value. Taking the popular outdoor positioning system, GPS, as an example, many practical positioning applications have been developed. However, GPS performs too poorly inside buildings to provide usable indoor positioning, thus, indoor environment need other positioning technologies to obtain position information.

\section{Wi-Fi Fingerprint Location}

The fingerprint technique contains two phases [3]: the offline phase and the online phase. The entire process is shown in Figure 1.During the offline phase, the method uses the mobile phone to construct an offline signal map by collecting information about Wi-Fi signal strength for a large number of positions. During the online phase, this algorithm goes through the database and picks the $\mathrm{k}$ referenced positions that match best received signal strength tuple. The criterion that is commonly retained is the Euclidian distance metric.

The main advantage of this method is its simplicity to set it up. However the accuracy highly depends on the granularity of the reference database. A better accuracy can be achieved with finer grids, but a finer grids means a larger database that is more time costly. 


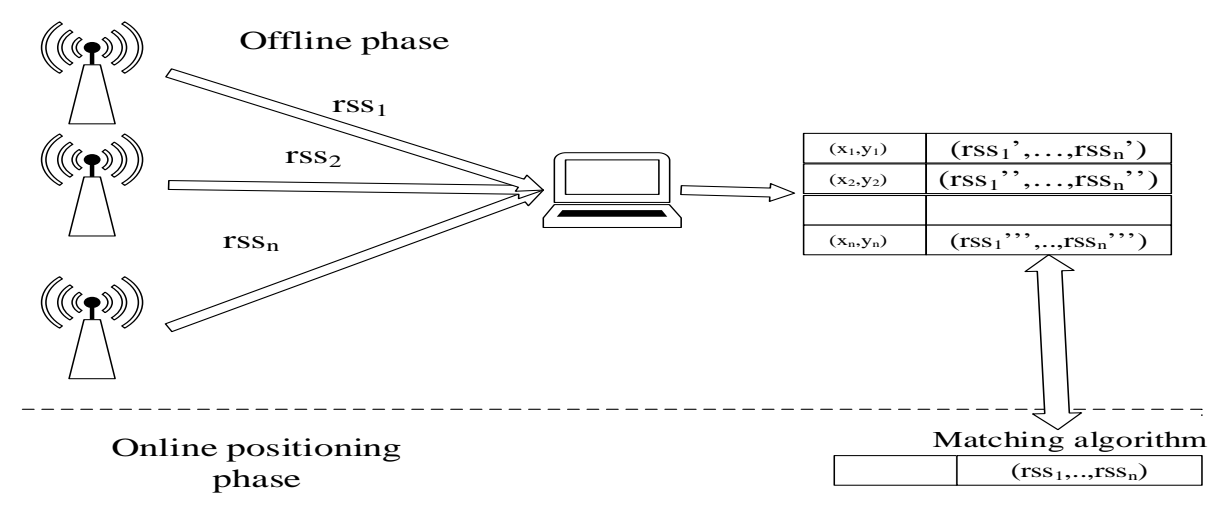

Fig.1. Diagram of fingerprint location

\section{Inertial Navigation and Positioning based on Motion Inertial Sensors}

INSs are self-contained, dead-reckoning navigation systems, which provide dynamic information through direct measurements.

In this experiment, the available sensors are: a gyroscope that delivers some information about the angular speed of the mobile; an accelerometer that delivers acceleration sensor data about the mobile. Through reckoning algorithm and cooperation of accelerometer and gyroscope, we can obtain three-dimensional acceleration and three-dimensional velocity of equipment [4].

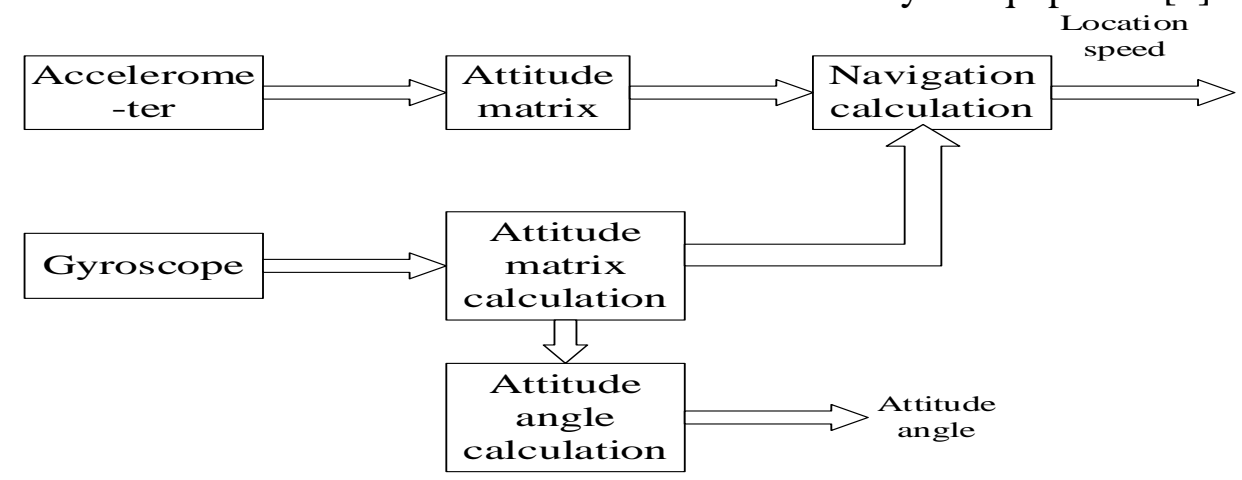

Fig.2. Diagram of motion sensor

Actual motion acceleration of carrier can be obtained by projecting acceleration on the motion direction. Finally, quadratic integral of acceleration information is designed from the time domain to get the carrier's distance on the motion direction.

Although the information rates are reliable over long periods of time, they must be integrated to provide orientation, linear position, and velocity information. Thus, even very small errors in the information rates can cause an unbounded growth in the error of integrated measurement. One way of overcoming this problem is to use inertial sensors in conjunction with other absolute mechanisms to periodically reset them.

\section{Augmented Reality}

Based on the analysis of images that camera caught, augmented reality determines the position of the objects in the real scene shows. Firstly, some artificial markers are placed in the real scene, then the camera shoots them and stores them, they can be used to computer in the form of digital images. Computers analysis digital image to determine the existing areas of artificial marks and return location information hidden in the marks to the user.

\section{Ultrasonic positioning technology}

Ultrasonic positioning sets the real signal transmitters in the real scene, which makes the method not subject to regional restriction and the positioning system more stable. The signal transmitters belong to the indoor positioning device, which can lock the user in a small scale region and provide 
more precise location result for user. Ultrasonic positioning system framework as follows:

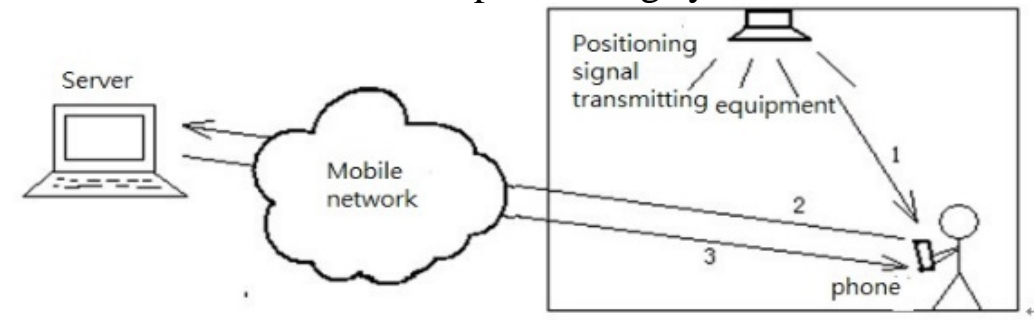

The working process:

Fig.3. Diagram of the framework

Step 1: When the user enters the ultrasonic coverage, the user's mobile phone detects the ultrasonic signal from the transmitter automatically and sends it to the specified server through the mobile network.

Step 2: After the server receives the position signal, via demodulation, the server can obtain the location information and send the information to the user through the mobile network.

Step 3: The mobile phone will complete positioning function through location information above.

\section{Multi-mode positioning system design}

In this section, we develop a framework for analyzing a simple and convenient indoor positioning system. Location information obtained by above four kinds of localization way can be fused to obtain a higher positioning performance, the positioning principle as shown:

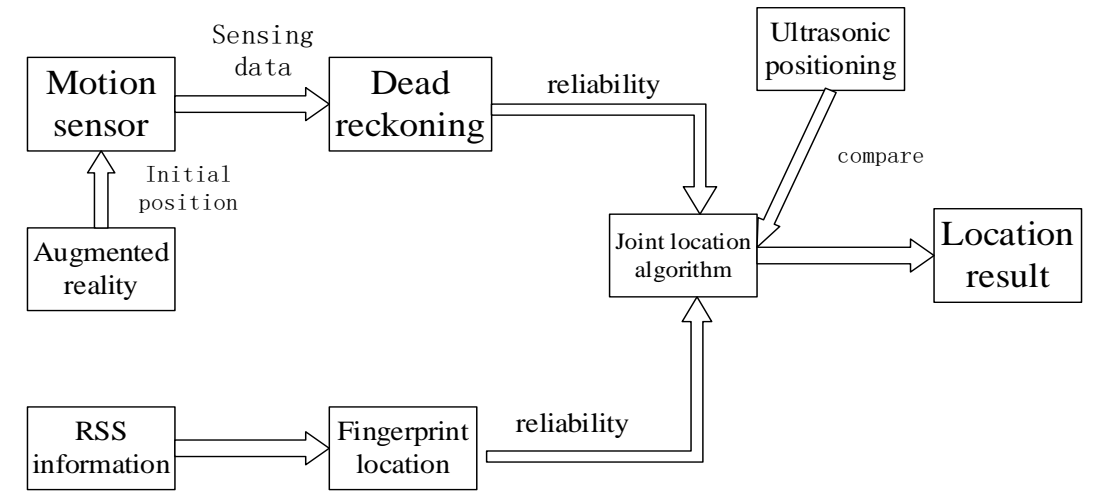

Fig.4.Diagram of joint location

In fingerprint location algorithm, if $\mathrm{Z}=\mathrm{R}\left(\mathrm{rss}_{1}, \ldots, \mathrm{rss}_{\mathrm{n}}\right)$ is the observed RSS vector composed of $\mathrm{n}$ received access points at the unknown position $\mathrm{X}=(\mathrm{x}, \mathrm{y})$ and $\mathrm{Zi}$ the footprint recorded in the database for the position $\mathrm{Xi}=\left(\mathrm{x}_{\mathrm{i}}, \mathrm{y}_{\mathrm{i}}\right)$, then this Euclidian distance is [5]:

$$
\mathrm{d}\left(\mathrm{Z}, \mathrm{Z}_{\mathrm{i}}\right)=\frac{1}{M} \sqrt{\sum_{j=1}^{M}\left(R S S_{j}(x, y)-R S S_{j}\left(x_{i}, y_{i}\right)\right)^{2}}
$$

Where $\mathrm{RSS}_{\mathrm{j}}\left(\mathrm{x}_{\mathrm{i}}, \mathrm{y}_{\mathrm{i}}\right)$ is the mean value recorded in the database for the access point whose MAC address is noted " $\mathrm{j}$ ” at the position $\left(\mathrm{x}_{\mathrm{i}}, \mathrm{y}_{\mathrm{i}}\right)$.

The set $\mathrm{N}_{\mathrm{k}}$ of the database positions is built with an interactive process as follows:

$$
\mathrm{N}_{k}=\left\{\operatorname{argmin}\left[d\left(Z, Z_{i}\right)\right] \backslash X \quad \notin N_{K-1} X_{i} \in \varepsilon\right\}
$$

Where $\varepsilon$ is the set of positions recorded in the database, this set contains k positions. Finally, the position of the mobile is considered to be the barycenter of those k selected positions.

$$
\mathrm{X}=\frac{\sum_{j=1}^{k}\left(\frac{1}{d\left(z, Z_{j}\right)}\right) \cdot X_{j}}{\sum_{j=1}^{k}\left(1 / d\left(Z, Z_{j}\right)\right)} \text { with } X_{j} \in N_{k}
$$

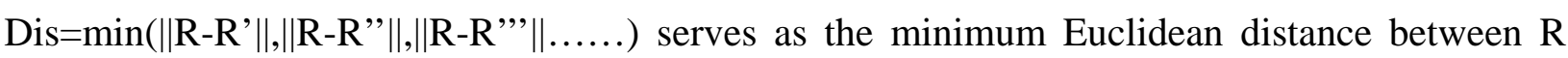


and the k points, the smaller the value of Dis, the higher the credibility of location results. When Dis is greater than a threshold value $\Theta$, the positioning result can be considered as invalid.

Using sensor data obtained from gyroscope and accelerometer, the quaternion algorithm can estimate the carrier attitude. Proceed as follows:

1. Initial attitude quaternion $(w, x, y, z)=(1,0,0,0)$ is named $A$.

2. Multiply the angular velocity from three-axis gyroscope by time interval to get Euler angel $b$.

3. Convert $b$ to quaternion $B$.

4. $A=A * B$, get the new quaternion corresponding to the current position.

5. Velocity calculation.

6. Displacement calculation

For the Wi-Fi positioning system presented above, the INS positioning is to get a better knowledge of the behavior of the mobile in order to reduce the effect of the Wi-Fi measurement noise and achieve real-time positioning when Wi-Fi signal occur big changes suddenly in indoor environment. We can merge the above two kinds of localization way [6]. Preset initial position can be set as the starting position of the SINS algorithm, in the process of movement, the localization algorithm based on reliability can obtain joint position location.

Reliability factor $\alpha \in[0,1]$ can be used to describe the reliability of fingerprinting. The relationship between Dis and $\alpha$ as shown in equation (4):

$$
\alpha= \begin{cases}1-\frac{1}{\theta} \text { Dis } & \text { Dis }<\theta \\ 0 & \text { Dis } \geq \theta\end{cases}
$$

When navigation time is longer than $\mathrm{X}$ seconds, the positioning error is too large, positioning result can be considered as unreliable result. Reliability factor $\beta \in[0,1]$ can be used to describe the reliability of inertial navigation system, the value decrease with time in slope value $1 / x$, its relationship with time as shown in equation (5):

$$
\beta= \begin{cases}1-\frac{1}{x} t & t<x \\ 0 & t \geq x\end{cases}
$$

In the above formula, after $\mathrm{x}$ seconds, the reliability of the SINS system becomes 0 , it makes no contribution to the final positioning result.

Final positioning result obtained by the following formula:

loc $=\frac{\alpha}{\alpha+\beta} \operatorname{loc}_{1}+\frac{\beta}{\alpha+\beta} \operatorname{loc}_{2}$

loc indicates the final estimated position, $\operatorname{loc}_{1}$ indicates position estimated by fingerprinting location, $\operatorname{loc}_{2}$ indicates result estimated by SINS.

In practice, the acquisition cycle of Fingerprinting is T1, the acquisition cycle of motion sensor is T2. Typically T1>T2, and in general, there is a plurality of continuous motion sensing information within an RSS acquisition cycle. Therefore location information is fused by formula on each T1 cycle time, the fusion result is used as an initial position of next round.

Trajectory obtained by above method will show fluctuation due to a variety of measurement error in real-time positioning process. Even very small errors in the information rates can cause an unbounded growth in the error of integrated measurements.

But in real life, multi-story building has become very common, fluctuation may lead to the error of floor judgement, if users want to get accurate indoor positioning, firstly accurate floor location is a must. In view of the above situation, we can install ultrasonic nodes at each floor. When the user enters the coverage area covered by ultrasound signal, coordinate information can be demodulated from the ultrasonic signal, which can be used to obtain accurate floor information.

In addition, coordinate information demodulated from ultrasonic signal and coordinate information obtained from the joint positioning can conduct distance calculation, if the calculated result exceeds the coverage radius of the ultrasonic signal, the positioning result adopts coordinate information demodulated from ultrasonic. Instead, the result does not change. The method can avoid the serious positioning error and reduce positioning error to less than $\mathrm{R} \mathrm{m}$. 
But as time goes on, the error of inertial navigation system will increase. One way of overcoming this problem is to use above method in conjunction with other positioning method to reset them.

Image matching technology can clear all the positioning error, which also can be used to reset the inertial sensors. If the user enables augmented reality location in augmented reality nodes, error is cleared and initial location is updated, its reliability is 1.

Moreover, some users may be make restrictions. For example, specific equipment can only start at specific point. Wi-Fi positioning can still play its role, to ensure the system stability.

The next section presents the results that are obtained by using all these techniques.

\section{Program Verification}

Experimentations were conducted to get a better idea of the performances that can be awaited from such positioning techniques. Experimentations were carried out on the second floor of a university teaching building. The mobile terminal was a laptop on which all algorithm were running. The signal strength in signal database built with a measurement every two floor squares in the corridor. Some real measurement are collected along this path and then reused to estimate the performance of the technique.

The article built verification platform on Matlab, which achieved positioning algorithm. By contrast of single-mode positioning effect and fusing positioning effect, the program showed the superiority. The collection of training data is implemented on mobile device, which through an application called Wi-Fi analyzer.

The actual floor area collected 91 node signals, eight nodes completed coverage. The experiment did not need coordinates of AP nodes in advance. The distribution of reference nodes as shown in figure 6.

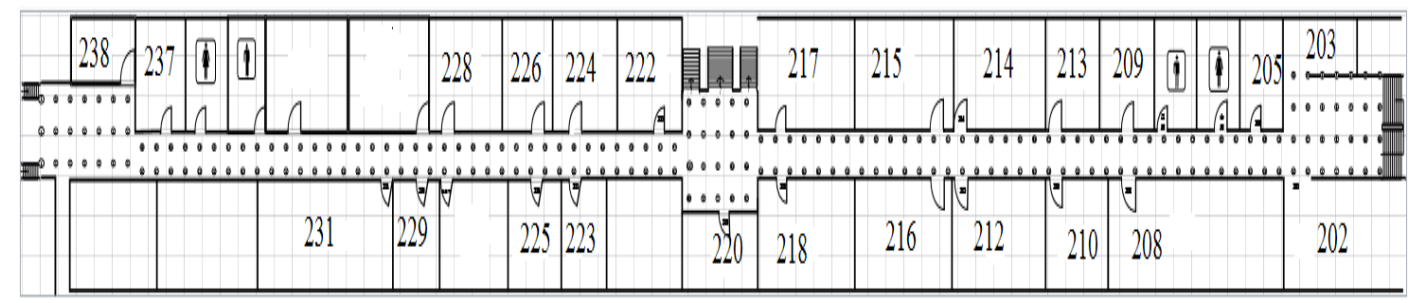

Fig.6.The distribution of sample nodes

A first experiment was carried out to show the performance of the fingerprinting algorithm presented in above. Firstly, ultrasonic positioning accomplished floor judgement. The paper selected a path in test environment as shown in figure 7. The coordinate of label points have already been known, by analyzing them and the coordinates generated by Wi-Fi positioning, positioning error could be calculated.

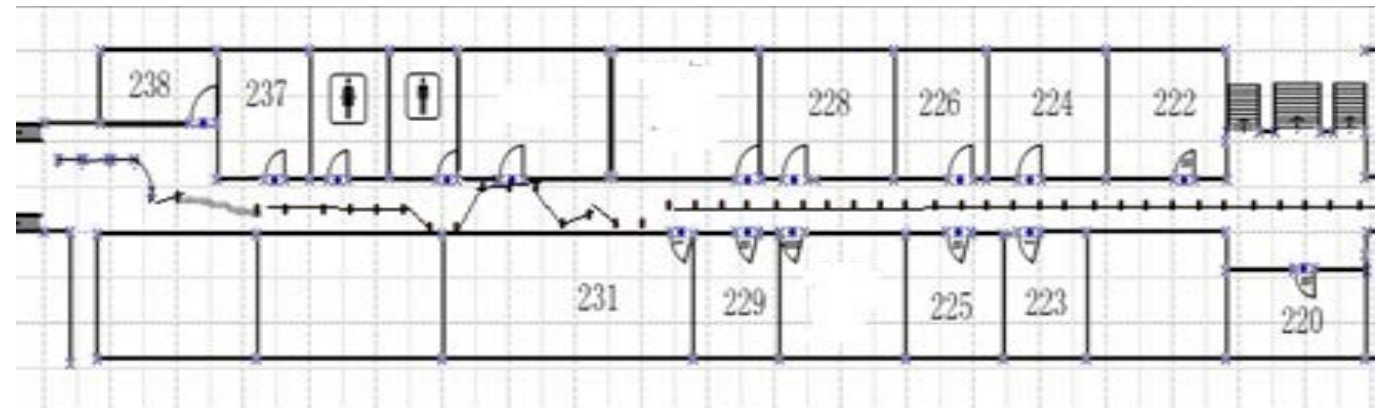

Fig.7.Diagram of location route

The figure 8 showed the result of Wi-Fi positioning. The result of fingerprint algorithm existed error of about $3 \mathrm{~m}$, the maximum error can even reach $8 \mathrm{~m}$. The number of nodes that positioning error less than $3 \mathrm{~m}$ were $58.3 \%$, the number of nodes that positioning error greater than $5 \mathrm{~m}$ were 
$13 \%$.

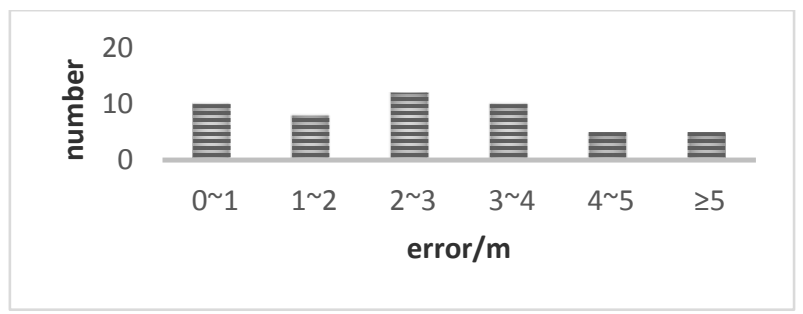

Fig.8.The result of Wi-Fi positioning

In contrast experiment, their position were estimated by s multi-mode fusion algorithm. Firstly, ultrasonic positioning accomplished floor judgement. The direction of the arrow indicated if the estimation was delayed or advanced in comparison to the real position of the mobile.

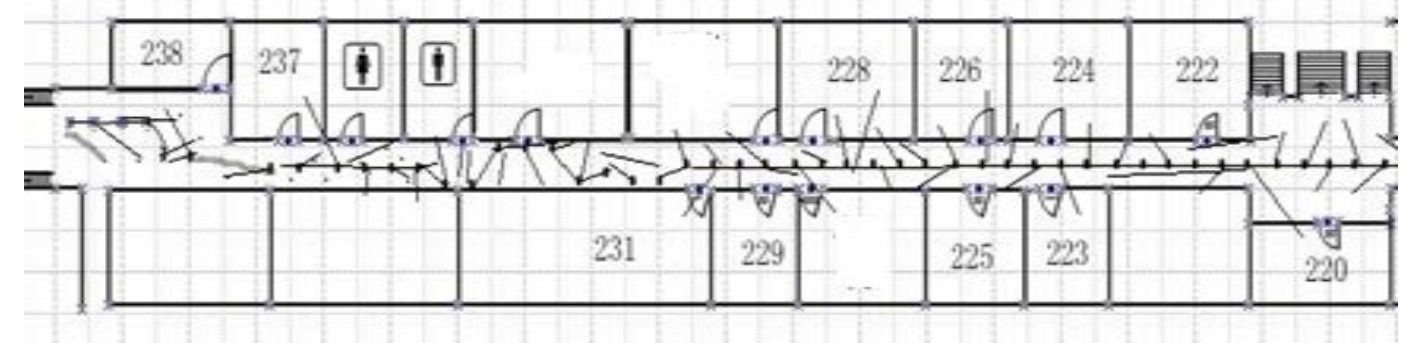

Fig.9.The result of joint positioning

The above analysis had obtained that the result of fingerprint matching algorithm existed error of about $3 \mathrm{~m}$, the maximum error can even reach $8 \mathrm{~m}$. It could only locate the target in the position of training samples, so its result was easily influenced by density of training samples.

Through the analysis of the data, the number of nodes that positioning error less than $3 \mathrm{~m}$ were $88.8 \%$, greater than $58.3 \%$, the number of nodes that error greater than $5 \mathrm{~m}$ were $0.5 \%$, less than $13 \%$, we could see from above figure, some nodes that used augmented reality could clear error and reseted INS, so the average error of fusion position was less than $2 \mathrm{~m}$, which verified the effectiveness of the method.

\section{Conclusion}

Regarding to the performances that are awaited from the technology, different techniques can be applied. For the most complex one, fusing information from the Wi-Fi network, INS, ultrasonic, with information coming from augmented reality, it is possible to get performances close to the meter accuracy. As the function of mobile terminal is getting stronger and stronger, such a positioning system should be available in the coming years.

\section{Acknowledgement}

The work presented in this paper was supported by the National Great Science Specific Project (Grants No. 2014ZX03002002-004) and National Natural Science Foundation of China (Grants No. NSFC-61471067).

\section{References}

[1] M. KLANN, "Playing with fire: User-centered design of wearable computing for emergency response,” in Proc. Mobile Response '07, 2007, pp. 116-125

[2] I. CONSTANDACHE, R. R. Choudhury, and I. Rhee, "Towards mobile phone localization without war-driving,” in Proc. INFOCOM '10, 2010, pp. 1-9.

[3] YAO Q M, WANG F Y, GAO H, et al. Location estimation in ZigBee network based on fingerprinting [C]. IEEE International Conference on Vehicular Electronics and Safety, ICVES, 2007. 
[4] KUSHKI A, PLATANIOTIS L N.VENETSANOPOULOS A N. Kernel-based positioning in wireless local area networks [J].IEEE Transactions on Mobile Computing, 2007, 6(6):689-705.

[5] OUTEMZABET S, NERGYIZIAN C. Accuracy enhancement of an indoor ANN-based fingerprinting location system using particle filtering and a low-cost sensor [C]. IEEE Vehicular Technology Conference, 2008: 2750-2754.

[6] Frederic and Francois Marx: Advanced integration of wifi and inertial navigation systems for indoor mobile positioning. EURASIP Journal on Applied Signal Processing Volume 2006. 